\title{
PROBABILITY DENSITY EVOLUTION ANALYSIS OF SEISMIC RESPONSE OF NONLINEAR STRUCTURES WITH RANDOM PARAMETERS
}

\author{
Junyi Yang ${ }^{1}$, Jianbing Chen ${ }^{2}$, and $\mathrm{Jie} \mathrm{Li}^{3}$ \\ ${ }^{1} \mathrm{Ph}$. D. Student \\ School of Civil Engineering, Tongji University, Shanghai 200092, China \\ e-mail: yangjunyistill@163.com \\ ${ }^{2}$ Professor
}

State Key Laboratory of Disaster Reduction in Civil Engineering, Tongji University, Shanghai 200092, China

chenjb@tongji.edu.cn

${ }^{3}$ Distinguished Professor

State Key Laboratory of Disaster Reduction in Civil Engineering, Tongji University, Shanghai 200092, China

lijie@tongji.edu.cn

Keywords: Stochastic Nonlinear Seismic Responses, Reliability, Generalized F-discrepancy, Probability Density Evolution Method (PDEM).

\begin{abstract}
The performance and reliability of engineering structures under extreme disastrous dynamic excitations (e.g. earthquakes) is of great concern due to the strong nonlinear mechanical behaviors as well as the uncertainty involved in both structural properties and external excitations. Great efforts have been devoted into this field in the past over half a century, yielding a variety of approaches, e.g, the Monte Carlo simulation and the improvements, the perturbation method and the orthogonal polynomials expansion method, ect. However, it remains to be a great challenge to obtain the probabilistic information of the stochastic seismic responses of nonlinear engineering structures. The development of probability density evolution method (PDEM) based on the principle of preservation of probability provides a new approach to this problem. In the present paper, a new generalized $F$-discrepancy (GF-discrepancy) is adopted in the strategy of selection of representative points so that the efficiency and accuracy of PDEM could be improved and guaranteed. Then the stochastic seismic response of a nonlinear frame-shear structure with random parameters is analyzed, and the reliabilities under different threshold levels are estimated. Problems to be further studied are outlined.
\end{abstract}




\section{INTRODUCTION}

Due to the randomness of the time and magnitude of earthquakes, it is possible for all the practical engineering structures to enter the phase of exhibiting nonlinear behaviors $[1,2]$. Therefore, it is of great importance to take into account the coupling of nonlinearity and randomness in assessing the performance and reliability of a practical structure rationally. In the past few decades, though the stochastic dynamics of structures, including the random vibration and the stochastic structural analysis, was extensively investigated, yielding a variety of approaches such as the Monte Carlo simulation (MCS) [3], the random perturbation method [4, 5], and the orthogonal polynomials expansion [6,7], ect., great challenges are still encountered in the stochastic dynamical analysis of nonlinear structures of practical engineering interest [8]. Moreover, these methods are mainly aimed at obtaining the first second order statistics of structural responses rather than higher order statistics or probability density functions, which are of crucial importance in the reliability evaluation of nonlinear structures $[9,10]$. From the perspective of physical stochastic systems, a family of probability density evolution method (PDEM), which is capable of capturing the instantaneous probability density functions of stochastic responses of nonlinear structures, has been developed by $\mathrm{Li}$ and Chen in the past decade [11]. Based on the random event description of principle of preservation of probability, a generalized density evolution equation (GDEE), of which the dimension is free from the dimension of the original dynamical system, is derived and solved by incorporating the embedded physical equations [12].

Two possible paths, i.e., the ensemble evolution path and the point evolution path, could be employed in the numerical solution of PDEM [12]. In the point evolution based approach, a smart selection of representative points is of paramount importance. Great endeavors have been devoted into the optimal selection of representative point set $[13,14]$. In the present paper, the generalized F-discrepancy (GF-discrepancy) is employed in the determination of representative points. A numerical example of a nonlinear frame-shear structure is presented. The stochastic seismic responses and the corresponding probabilistic information, including probability density functions, are obtained and analyzed. Then the evaluation of reliabilities under different threshold levels are carried out so that the accuracy and efficiency of PDEM could be verified. Problems to be further studied are discussed.

\section{FUNDAMENTALS OF PROBABILITY DENSITY EVOLUTION METHOD (PDEM)}

Without loss of generality, the equation of motion of a multi-degree-of-freedom (MDOF) structure reads

$$
\mathbf{M}(\boldsymbol{\Theta}) \ddot{\mathbf{X}}+\mathbf{C}(\boldsymbol{\Theta}) \dot{\mathbf{X}}+\mathbf{f}(\boldsymbol{\Theta}, \mathbf{X})=\mathbf{\Gamma G}(\boldsymbol{\Theta}, t)
$$

where $\mathbf{M}$ and $\mathbf{C}$ are the $n$ by $n$ mass and damping matrices, respectively; $\mathbf{f}$ is the $n$ by 1 linear or nonlinear restoring force vector; $\mathbf{X}, \dot{\mathbf{X}}$ and $\ddot{\mathbf{X}}$ are the $n$ by 1 displacement, velocity and acceleration vectors, respectively; $\boldsymbol{\Gamma}$ is the $n$ by $r$ loading influence matrix and $\mathbf{G}$ is the $r$ by 1 random loading vector; $\boldsymbol{\Theta}$ denotes the 
$s$-dimensional basic random vector with known joint $\operatorname{PDF} p_{\Theta}(\boldsymbol{\theta})$ containing all the basic random variables involved both in structural parameters and earthquake excitations.

For a well-posed dynamical system, the solution of Eq. (1) exists as the function of $\boldsymbol{\Theta}$, and must be unique. However, in order to assess the performance and the reliability of a practical structure under disastrous external excitations, some other physical quantities such as the internal forces (e.g., moments, axial forces and shearing forces) at crucial sections, the stress or the strain at key points and so on, are of engineering interest. According to the geometric consistency rules and the constitutive laws, these quantities can be obtained once the displacement and the velocity are known. Therefore, the other physical quantities of the system, which are denoted by $\mathbf{Z}(t)=\left(Z_{1}(t), Z_{2}(t), \ldots, Z_{m}(t)\right)$, must be the function of $\boldsymbol{\Theta}$. Undoubtedly, the derivative of $\mathbf{Z}(t)$, i.e. the generalized velocity, is also a function of $\boldsymbol{\Theta}$. Therefore, it is reasonable to assume that

$$
\begin{gathered}
\mathbf{Z}=\mathbf{H}_{\mathbf{Z}}(\boldsymbol{\Theta}, t) \\
\dot{\mathbf{Z}}(t)=\mathbf{h}_{\mathbf{Z}}(\boldsymbol{\Theta}, t)
\end{gathered}
$$

where $\mathbf{H}_{\mathbf{z}}=\left(\mathrm{H}_{\mathbf{z}, 1}, \mathrm{H}_{\mathbf{z}, 2}, \cdots, \mathrm{H}_{\mathbf{z}, m}\right), \quad \mathbf{h}_{\mathbf{z}}=\left(h_{\mathbf{z}, 1}, h_{\mathbf{z}, 2}, \cdots, h_{\mathbf{z}, m}\right)$ and $m$ is the number of quantities of interest.

According to the random event description of the principle of preservation of probability [15], we have

$$
\frac{d}{d t} \int_{\Omega_{t} \times \Omega_{\theta}} p_{\mathbf{z} \boldsymbol{\Theta}}(\mathbf{z}, \boldsymbol{\theta}, t) d \mathbf{z} d \boldsymbol{\theta}=0
$$

where $p_{\mathbf{z} \boldsymbol{\Theta}}(\mathbf{z}, \boldsymbol{\theta}, t)$ is the joint PDF of $(\mathbf{Z}(t), \boldsymbol{\Theta}), \Omega_{\theta}$ is any arbitrary sub-domain belongs to $\Omega_{\Theta}$, the distribution domain of the random vector, i.e., the support of the joint $\operatorname{PDF} p_{\Theta}(\boldsymbol{\theta}), \Omega_{t}$ is the subdomain at time $t$ corresponding to an any arbitrary subdomain $\Omega_{0}$ at the initial time belongs to the distribution domain of $\mathbf{Z}(t)$.

After some mathematical manipulations, we can finally have a multi-dimensional integrodifferential equation

$$
\int_{\Omega_{\theta}}\left(\frac{\partial p_{\mathbf{z} \boldsymbol{\Theta}}(\mathbf{z}, \boldsymbol{\theta}, t)}{\partial t}+\sum_{i=1}^{m} \dot{Z}_{i}(\boldsymbol{\theta}, t) \frac{\partial p_{\mathbf{z} \boldsymbol{\Theta}}(\mathbf{z}, \boldsymbol{\theta}, t)}{\partial z_{i}}\right) d \boldsymbol{\theta}=0
$$

Mathematically equivalently, we have the following partial differential equation $[12,15]$, which governs the evolution of the joint PDF $p_{\mathbf{z \Theta}}(\mathbf{z}, \boldsymbol{\theta}, t)$

$$
\frac{\partial p_{\mathbf{z \Theta}}(\mathbf{z}, \boldsymbol{\theta}, t)}{\partial t}+\sum_{i=1}^{m} \dot{Z}_{i}(\boldsymbol{\theta}, t) \frac{\partial p_{\mathbf{z \Theta}}(\mathbf{z}, \boldsymbol{\theta}, t)}{\partial z_{i}}=0
$$

Apparently, in the case of $m=1$, Eq. (4) reduces to a one-dimensional partial differential equation, i.e.

$$
\frac{\partial p_{z \boldsymbol{\Theta}}(z, \boldsymbol{\theta}, t)}{\partial t}+\dot{Z}(\boldsymbol{\theta}, t) \frac{\partial p_{z \boldsymbol{\Theta}}(z, \boldsymbol{\theta}, t)}{\partial z}=0
$$

Eqs. (4) and (5) are the so-called generalized density evolution equations (GDEEs) $[12,15]$ and the corresponding initial condition of Eq.(5) could be 


$$
\left.p_{\mathrm{Z} \boldsymbol{\Theta}}(z, \boldsymbol{\theta}, t)\right|_{t=t_{0}}=\delta\left(z-\mathrm{z}_{0}\right) p_{\boldsymbol{\Theta}}(\boldsymbol{\theta})
$$

where $\mathrm{z}_{0}$ is the deterministic initial value and $\delta(\cdot)$ is Dirac's delta function.

By solving Eq.(5), the instantaneous PDF of $Z(t)$ could be obtained by

$$
p_{\mathrm{Z}}(z, t)=\int_{\Omega_{\Theta}} p_{\mathrm{Z} \boldsymbol{\Theta}}(z, \boldsymbol{\theta}, t) d \boldsymbol{\theta}
$$

in which $p_{Z}(z, t)$ denotes the PDF of $Z(t)$.

It is also noted that Eq.(3) holds for any arbitrary subdomain $\Omega_{\theta}$. If we partition the domain $\Omega_{\Theta}$ into exclusive subdomains $\Omega_{q}, q=1,2, \cdots, n$, i.e., $\bigcup_{q=1}^{n} \Omega_{q}=\Omega_{\Theta}$, and $\Omega_{r} \cap \Omega_{q}=\varnothing, \forall r \neq q$, then Eq.(3) becomes

$$
\frac{\partial p_{q}(\mathbf{z}, t)}{\partial t}+\sum_{i=1}^{m} \frac{\partial J_{q, i}(\mathbf{z}, t)}{\partial z_{i}}=0
$$

where $J_{q, i}(\mathbf{z}, t)=\int_{\Omega_{q}} \dot{Z}_{i}(\boldsymbol{\theta}, t) p_{\mathbf{z \Theta}}(\mathbf{z}, \boldsymbol{\theta}, t) d \boldsymbol{\theta}$ is the sub-flux of probability in the $i$-th direction, and $p_{q}(\mathbf{z}, t)=\int_{\Omega_{q}} p_{\mathbf{z \Theta}}(\mathbf{z}, \boldsymbol{\theta}, t) d \boldsymbol{\theta}$. Clearly, Eq.(7) is also a conservative type equation, which is in turn the embodiment of the principle of preservation of probability. In this form, Eq.(7) becomes $p_{\mathbf{z}}(\mathbf{z}, t)=\sum_{q=1}^{n} p_{q}(\mathbf{z}, t)$. It is noted that Eq.(7) is a form of ensemble evolution type equation.

It should be emphasized that, even for a multi-dimensional problem, a one-dimensional differential equation could be derived and solved in PDEM. Therefore, the PDEM provides a feasible way to implement stochastic dynamical analysis of generic MDOF systems, which remains to be a great challenge for the classical equations (e.g. the Liouville equation, the FPK equation and the Dostupove-Pugachev equation) [16].

\section{THE GENERALIZED F-DISCREPANCY (GF-DISCREPANCY)}

The direct solution based on the ensemble evolution type equation (7) is still to be developed. However, some advances have been made based on this thought. For instance, the FPK equation could be reduced to a lower-dimensional partial differential equation [17].

On the other hand, Eq. (7) could be reduced to a set of point evolution based equations

$$
\frac{\partial p_{Z \Theta}\left(z, \boldsymbol{\theta}_{q}, t\right)}{\partial t}+\dot{Z}\left(\boldsymbol{\theta}_{q}, t\right) \frac{\partial p_{Z \Theta}\left(z, \boldsymbol{\theta}_{q}, t\right)}{\partial z}=0, \quad q=1,2, \cdots, n
$$

where the representative points $\mathcal{M}_{n}=\left\{\boldsymbol{\theta}_{q}=\left(\theta_{1, q}, \theta_{2, q}, \cdots, \theta_{s, q}\right) \in \Omega_{q} \mid q=1,2, \cdots, n\right\}$ are selected and the corresponding assigned probabilities are calculated as the following equation $[12,18]$ so that the probabilistic information of the subdomain $\Omega_{q}$ could be represented by $\boldsymbol{\theta}_{q}$, i.e.

$$
P_{q}=\int_{\Omega_{q}} p_{\Theta}(\boldsymbol{\theta}) d \boldsymbol{\theta}
$$

$\dot{Z}\left(\boldsymbol{\theta}_{q}, \mathrm{t}\right)$ in Eq.(7) could be obtained by solving Eqs. (1) and (2). 
By adopting appropriate finite difference scheme (e.g. Lax-Wendroff scheme or TVD scheme), Eqs. (7)错误!未找到引用源。 could be solved and then the instantaneous PDF of $Z(t)$ could be evaluated as

$$
p_{Z}(z, t)=\sum_{q=1}^{n} p_{z \boldsymbol{\Theta}}\left(z, \boldsymbol{\theta}_{q}, t\right)
$$

It is obvious that a smart selection of representative points is of paramount importance to achieve the tradeoffs between the accuracy and efficiency of the numerical solution of PDEM. In the present paper, the generalized F-discrepancy is adopted in the strategy of selecting representative points. The GF-discrepancy is defined as [14]

$$
D_{G F}\left(\mathcal{M}_{n}\right)=\max _{1 \leq i \leq m}\left\{\sup _{-\infty<\theta<\infty}\left|F_{n, i}(\theta)-F_{i}(\theta)\right|\right\}
$$

in which $F_{i}(\theta)$ is the cumulative distribution function (CDF) of the $i$-th dimension and $F_{n, i}(\theta)$ is the corresponding empirical CDF evaluated as

$$
F_{n, i}(\theta)=\sum_{q=1}^{n} P_{q} \cdot I\left\{\theta_{i, q}<\theta\right\}
$$

where $\theta_{i, q}$ denotes the coordinate of $\boldsymbol{\theta}_{q}$ along the $i$-th dimension, $P_{q}$ is the assigned probability defined in Eq. (8) and $I\{\}$ is the indicator function.

It has been verified that the representative point set with smaller GF-discrepancy could guarantee a more accurate and efficient solution of PDEM [14, 19]. Therefore, minimizing the GF-discrepancy of representative points could be employed as the criterion of selecting representative points.

\section{NUMERICAL EXAMPLE}

Consider a 30-story frame-shear structure subjected to scaled El-Centro (N-S) earthquake acceleration. The mean values of masses from bottom to top are 3.3, 3.3, 3.0, 3.0, 3.0, 3.0, 3.0, 2.8, 2.8, 2.8, 2.8, 2.8, 2.6, 2.6, 2.6, 2.6, 2.6, 2.4 2.4 2.4, 2.4, 2.4, $2.2,2.2,2.2,2.2,2.2,2.0,2.0$, and $2.0\left(\times 10^{5} \mathrm{~kg}\right)$, respectively. The initial lateral inter-story stiffness from bottom to top are 4.0, 4.0, 3.8, 3.8, 3.8, 3.8, 3.8, 3.6, 3.6, 3.6, 3.6, 3.6, 3.4, 3.4, 3.4, 3.4, 3.4, 3.2, 3.2, 3.2, 3.2, 3.2, 3.0, 3.0, 3.0, 3.0, 3.0, 2.8, and 2.8 $\left(\times 10^{5} \mathrm{kN} / \mathrm{m}\right)$ in turn. The masses are lognormally distributed and the inter-story stiffness follows the Weibull distributions. Therefore, there are 60 random variables involved in the system. The coefficients of variation of all the random variables are 0.2. The Rayleigh damping is adopted such that $\mathbf{C}=a \mathbf{M}+b \mathbf{K}$, where $\mathbf{C}, \mathbf{M}$ and $\mathbf{K}$ are the damping, mass and stiffness matrices, respectively, and $a=0.160 \mathrm{~s}^{-1}, b=0.012 \mathrm{~s}$. To capture the nonlinearity of the inter-story restoring force, the extended Bouc-Wen model is adopted [20]. All the thirteen parameters involved are $\alpha=0.04, A=1.0, n=$ $1.0, q=0.3, p=10, \lambda=0.5, \phi=0.05, \zeta=0.1, d_{\phi}=5.0, d_{v}=1.0, d_{\eta}=1.0, \beta=30$, and $\gamma$ $=250$, respectively. Attention is focused on the inter-story drift of first floor. A typical hysteretic curve of the first floor is shown in Figure 1. Clearly, strong nonlinearity is involved.

By the point rearrangement strategy based on reducing GF-discrepancy, 512 representative points are selected and PDEM is implemented to obtain the mean and standard deviation of nonlinear seismic responses. Monte Carlo simulation (MCS) with 100000 pseudo random points are carried out to verify the accuracy of PDEM. 
Figure 2 shows that the mean value and standard deviation of the first inter-story drift obtained by PDEM are almost identical to those obtained by MCS.

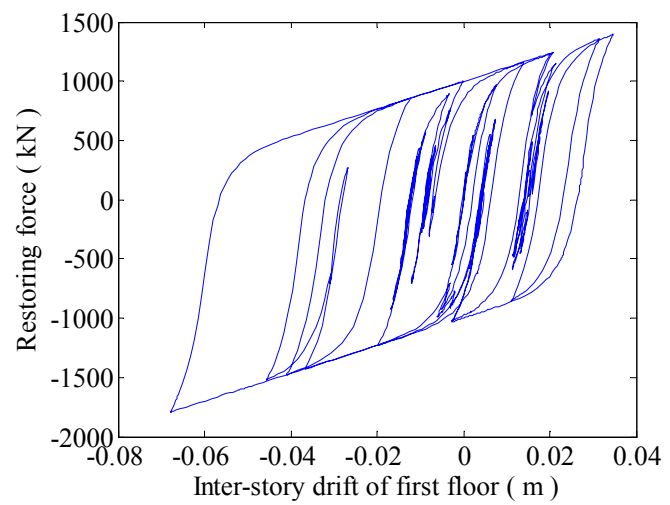

Figure 1: Typical hysteretic curve
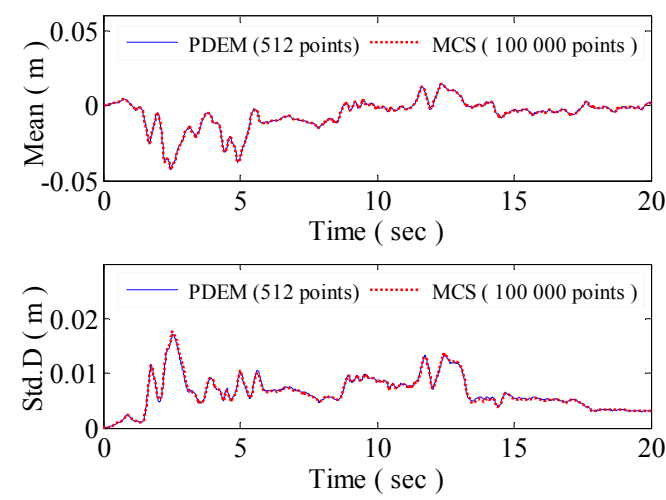

Figure 2: The mean and standard deviation

In order to verify the feasibility of minimizing GF-discrepancy as the criterion of selecting representative points, 10 times of MCS with 512 pseudo random points without GF-discrepancy-based optimization are carried out. The relative errors defined as the following equations are employed to exam the accuracy of PDEM with 512 representative points

$$
e_{\|\mu\|_{2}}=\frac{\left\|\mu_{1}-\mu_{0}\right\|_{2}}{\left\|\mu_{0}\right\|_{2}}, \quad e_{\|\sigma\|_{2}}=\frac{\left\|\sigma_{1}-\sigma_{0}\right\|_{2}}{\left\|\sigma_{0}\right\|_{2}}
$$

where $\mu_{0}$ and $\sigma_{0}$ denote the mean and standard deviation obtained by MCS (100000 points), respectively; $\mu_{1}$ and $\sigma_{0}$ denote those results obtained by PDEM (512 representative points) or those obtained by MCS (10 times of 512 points); $\|\cdot\|_{2}$ and $\|\cdot\|_{\infty}$ denote 2 - and infinite-norm, respectively. Table 1 shows the mean value, standard deviation, coefficient of variation and minimum value of the relative errors of PDEM and MCS (10 times of 512 points).

\begin{tabular}{ccccccc}
\hline & $\|\mu\|_{2}$ & $\|\sigma\|_{2}$ & $\|\mu\|_{\infty}$ & $\|\sigma\|_{\infty}$ & $D_{\mathrm{GF}}$ \\
\hline PDEM (512 representative points) & 0.0074 & 0.0252 & 0.0065 & 0.0416 & 0.0102 \\
\hline \multirow{3}{*}{$\begin{array}{c}\text { MCS } \\
\text { (512 points) }\end{array}$} & mean error & 0.0487 & 0.1653 & 0.0339 & 0.2007 & 0.1356 \\
\cline { 2 - 7 } & Std.D error & 0.0138 & 0.0552 & 0.0085 & 0.0768 & 0.0195 \\
\cline { 2 - 7 } & C.O.V & 0.2828 & 0.3160 & 0.2521 & 0.3827 & 0.1439 \\
\cline { 2 - 7 } & Minimum error & 0.0255 & 0.0838 & 0.0183 & 0.0865 & 0.1155 \\
\hline
\end{tabular}

Table 1: Comparison between PDEM and MCS (512)

It is shown in Table 1 that the representative point sets with GF-discrepancy-based optimization guarantee much more accurate results than those without GF-discrepancy-based optimization. Moreover, PDEM is capable of capturing any instantaneous PDF of nonlinear seismic response. Figure 3 shows 3 PDFs at 3 typical time instants and a remarkable evolution characteristic of PDF could be observed (e.g., there are three peaks at 8.77 second from single peak at 7.85 second, and then it turns out to be two peaks at 12.78 second). Figure 4 shows the corresponding CDFs at the 3 
typical time instants. It is obvious that the results of PDEM is in great accordance with those of MCS (100 000 points).

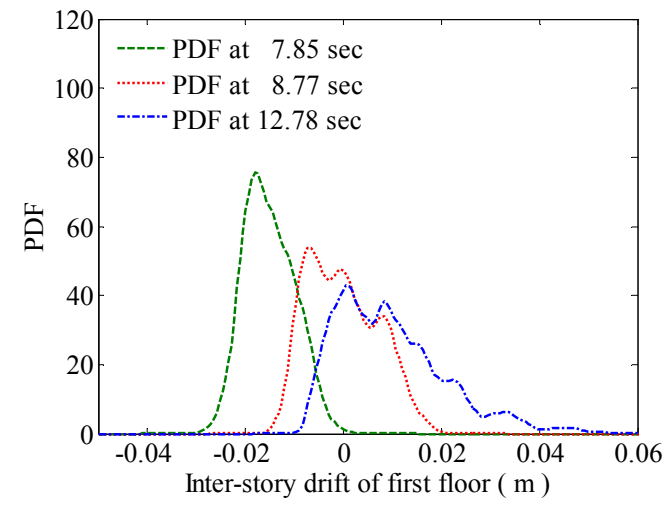

Figure 3: PDFs at three typical time instants

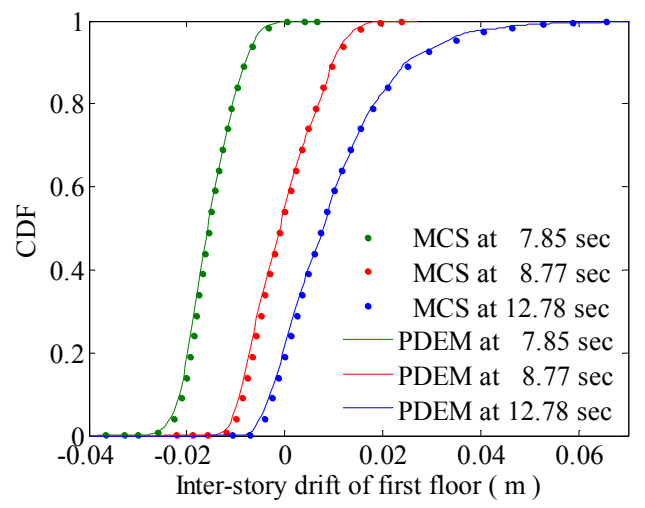

Figure 4: CDFs at three typical time instants

Figure 5(a) shows the evolution of PDF of the inter-story drift of first floor in a typical time interval. It could be observed that the evolution of PDF of nonlinear seismic responses is complicated and ever-changing. Thus the full access to the complete probabilistic information of nonlinear seismic responses, rather than the seconder order statistics obtained by the MCS, random perturbation method or the orthogonal polynomials expansion, is of critical importance in the assessment of safety and reliability of a practical engineering structure subjected to earthquake acceleration. In addition to capturing the evolution of PDF, PDEM is capable of evaluating the reliability of a structure under different threshold levels for nonlinear seismic responses. Based on the idea of equivalent extreme-value event and the extreme value distribution (EVD) [21, 22], CDF of the extreme value of inter-story drift of first floor, as shown in Figure 5(b), is obtained by the PDEM. It is shown in Figure 5(b) that the CDF of extreme value obtained by PDEM accords with that obtained by MCS (100000 points) very well. According to the CDF of extreme value shown in Figure 5(b), the failure probability of first floor with respect to inter-story drift could be estimated and the comparison of results between PDEM (512 points) and MCS (100000 points) is presented in Table 2.

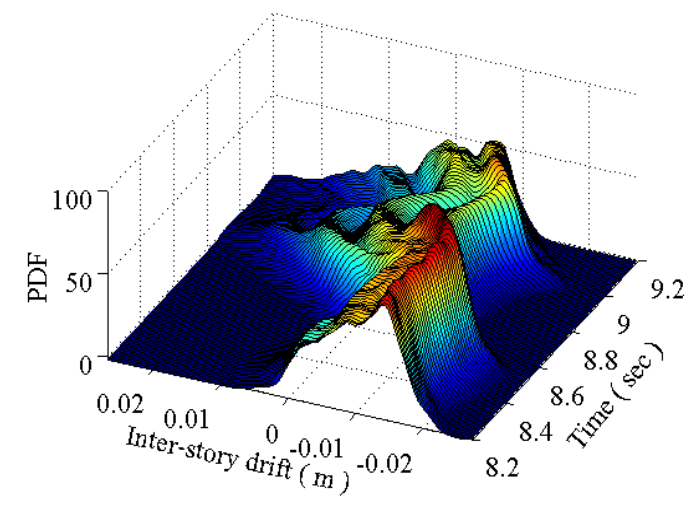

(a)

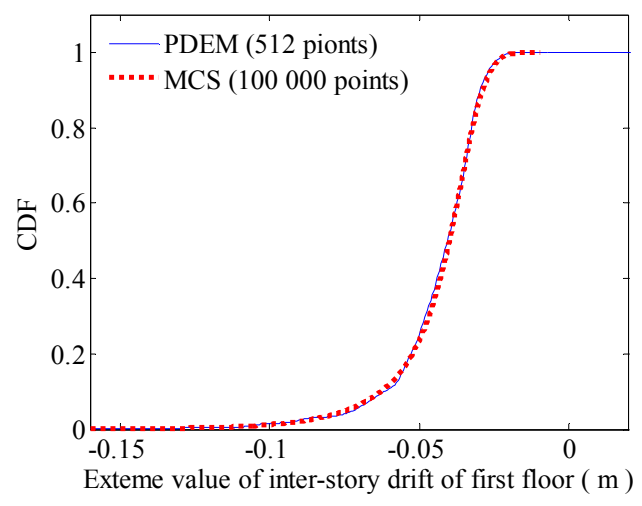

(b)

Figure 5: Analysis of probabilistic evolution and extreme value of nonlinear seismic response (a. Evolution of PDF of inter-story drift of first floor in a typical time interval; b. CDF of extreme value of inter-story drift of first floor) 


\begin{tabular}{ccccccc}
\hline \multicolumn{2}{c}{ Threshold level } & -0.08 & -0.10 & -0.12 & -0.14 & -0.16 \\
\hline \multirow{2}{*}{$P_{f}\left(\times 10^{-3}\right)$} & MCS & 36.04 & 12.23 & 4.54 & 1.71 & 0.59 \\
& PDEM & 36.91 & 15.71 & 4.82 & 1.74 & 0.11 \\
\hline
\end{tabular}

Table 2: Failure probability of first floor with respect to the inter-story drift under different threshold levels

The results presented in Table 2 demonstrate that PDEM guarantees enough accuracy when the failure probability is in the order of magnitude of $1.0 \times 10^{-3}$. More should be done if the probability of failure is much smaller to improve the accuracy.

\section{CONCLUSION}

In order to implement the assessment of the performance and reliability of a nonlinear structure under earthquake acceleration rationally, in which the coupling of nonlinearity and randomness is always involved, it is of great importance to obtain the complete probabilistic information of the stochastic seismic response of the nonlinear structure. The results of a numerical example of a nonlinear frame-shear structure demonstrate that PDEM is capable of capturing the instantaneous PDF of nonlinear seismic responses as well as the ever-changing evolution of PDF. By minimizing the GF-discrepancy in the selection of representative points, PDEM is able to achieve the tradeoffs between the accuracy and efficiency of the probability density evolution analysis as well as the evaluation of reliability of a structure under earthquake acceleration. Thus it is verified that GF-discrepancy could be employed in the strategy of selection of representative points. However, a better GF-discrepancy-based strategy of point selection is still to be explored when the probability of failure is extremely small.

\section{ACKNOWLEDGEMENTS}

Financial supports from the National Natural Science Foundation of China (NSFC Grant No.11172210 \& 51261120374) and the National Key Technology R \& D Program (Grant No. 2011BAJ09B03-02) are gratefully appreciated.

\section{REFERENCES}

[1] J. Li, G.Q. Li, Introduction to Earthquake Engineering. Beijing: Seismology Press, 1992. ( in Chinese )

[2] J.B. Chen, J. Li, Stochastic seismic response analysis of structures exhibiting high nonlinearity. Computers \& structures, 88(7), 395-412, 2010.

[3] M. Shinozuka, Monte Carlo solution of structural dynamics. Computers \& Structures, 2(5), 855-874, 1972.

[4] A.V. Skorokhod, F. Hoppensteadt, H. Salehi, Random perturbation methods with applications in science and engineering. Springer, 2002.

[5] M. Kleiber, T.D. Hien, The stochastic finite element method: basic perturbation technique and computer implementation. Wiley New York, 1992.

[6] J. Li, Stochastic structural system: Analysis and modeling. Beijing: Science Press, 1996. ( in Chinese ) 
[7] C.L. Wu, X.P. Ma, T. Fang, A complementary note on Gegenbauer polynomial approximation for random response problem of stochastic structure. Probabilistic Engineering Mechanics, 21(4), 410-419, 2006.

[8] B. Goller, H.J. Pradlwarter, G.I. Schueller, Reliability assessment in structural dynamics. Journal of Sound and Vibration, 332, 2488-2499, 2013.

[9] Y.G. Zhao, H. Idota, Response uncertainty and time-variant reliability analysis for hysteretic MDF structures. Earthquake Engineering and Structural Dynamics, 28, 1187-1213, 1999.

[10]J.B. Chen, J. Li, Development-process-of-nonlinearity-based reliability evaluation of structures. Probabilistic Engineering Mechanics, 22(3), 267-275, 2007.

[11]J. Li, J.B. Chen, Probability density evolution method for dynamic response analysis of structures with uncertain parameters. Computational Mechanics, 34(5), 400-409, 2004.

[12]J. Li, J.B. Chen, W.L. Sun, Y.B. Peng, Advances of the probability density evolution method for nonlinear stochastic systems. Probabilistic Engineering Mechanics, 28, 132-142, 2012.

[13]J. Xu, J.B. Chen, J. Li, Probability density evolution analysis of engineering structures via cubature points. Computational Mechanics, 50(1), 135-156, 2012.

[14]J.B. Chen, S.H. Zhang, Improving point selection in cubature by a new discrepancy. SIAM Journal on Scientific Computing, 35(5), A2121-A2149, 2013.

[15]J. Li, J.B. Chen, The principle of preservation of probability and the generalized density evolution equation. Structural Safety, 30(1), 65-77, 2008.

[16] J. Li, J.B. Chen, Stochastic dynamics of structures. John Wiley \& Sons, 2009.

[17]J.B. Chen, S.R. Yuan, PDEM-based dimension-reduction of FPK equation for additively excited hysteretic nonlinear systems. Probabilistic Engineering Mechanics, 38, 111-118, 2014.

[18] J.B. Chen, R. Ghanem, J. Li, Partition of the probability-assigned space in probability density evolution analysis of nonlinear stochastic structures. Probabilistic Engineering Mechanics, 24(1), 27-42, 2009.

[19]J.B. Chen, S.H. Zhang, Probability density evolution analysis of nonlinear response structures with non-uniform random parameters. Chinese Journal of Theoretical and Applied Mechanics, 46(1), 136-144, 2014. ( in Chinese )

[20]F. Ma, H. Zhang, A. Bockstedte, G.C. Foliente, P. Paevere, Parameter analysis of the differential model of hysteresis. Journal of Applied Mechanics, 71(3), 342-349, 2004.

[21] J. Li, J.B. Chen, W.L. Fan, The equivalent extreme-value event and evaluation of the structural system reliability. Structural Safety, 29(2), 112-131, 2007 
[22] J.B. Chen, J. Li, The extreme value distribution and dynamic reliability analysis of nonlinear structures with uncertain parameters. Structural Safety, 29(2), 77-93, 2007. 